\title{
Phase Transformation of Anisotropic Shape Memory Alloys: Theory and Validation in Superelasticity
}

\author{
Dimitris Chatziathanasiou ${ }^{1} \cdot$ Yves Chemisky $^{1} \cdot$ Fodil Meraghni $^{1}$. \\ George Chatzigeorgiou ${ }^{1} \cdot$ Etienne Patoor $^{1}$
}

Published online: 4 August 2015

(C) ASM International 2015

\begin{abstract}
In the present study, a new transformation criterion that includes the effect of tension-compression asymmetry and texture-induced anisotropy is proposed and combined with a thermodynamical model to describe the thermomechanical behavior of polycrystalline shape memory alloys. An altered Prager criterion has been developed, introducing a general transformation of the axes in the stress space. A convexity analysis of such criterion is included along with an identification strategy aimed at extracting the model parameters related to tension-compression asymmetry and anisotropy. These are identified from a numerical simulation of an SMA polycrystal, using a self-consistent micromechanical model previously developed by Patoor et al. (J Phys IV 6(C1):277-292, 1996) for several loading cases on isotropic, rolled, and drawn textures. Transformation surfaces in the stress and transformation strain spaces are obtained and compared with those predicted by the micromechanical model. The good agreement obtained between the macroscopic and the microscopic polycrystalline simulations states that the proposed criterion and transformation strain evolution equation can capture phenomenologically the effects of texture on anisotropy and asymmetry in SMAs.
\end{abstract}

Keywords Shape memory alloys - Superelasticity · Anisotropy - Tension-compression asymmetry · Transformation criteria $\cdot$ Evolution equation

Dimitris Chatziathanasiou

dimitrios.chatziathanasiou@ensam.eu

1 Arts et Métiers ParisTech, LEM3 UMR 7239 CNRS, 4 rue Augustin Fresnel, 57078 Metz - Cedex 01, France

\section{Introduction}

Shape memory alloys (SMAs) are metallic materials that can recover significantly large inelastic strains. When subjected to particular thermomechanical loadings, they can recuperate from strains that would be permanent for most common metals. The characteristic of large recoverable strains is the result of the transformation between the two key solid phases that the materials can adopt, austenite and martensite. The difference between these two phases lies on the architecture of the crystalline structure, which varies between a cubic-like configuration in austenite and a less-symmetric configuration in martensite. Forward transformation is defined as the transition from austenitic to martensitic phase, whereas the inverse procedure is defined as reverse transformation. The phase transformation is the result of either the change in temperature, if it varies between critical values, or the development of appropriate stress states within the material. In particular, applying a mechanical loading/unloading cycle above the transition temperatures results in the effect of superelasticity in SMAs. During forward transformation, the transformation starts at a critical, temperature-dependent stress. A strain plateau is observed in the uniaxial stress-strain diagram, before the start of the elastic section of martensite. In that case, the large strain that occurs between the two elastic sections on the stress-strain diagram corresponds to a transformation strain which is fully recovered when stress returns to zero.

Experimental characterization of the superelastic behavior of SMAs has shown that the material exhibits a tension-compression asymmetry [1,2]. Indeed, the critical stress for the onset of transformation is different between tension and compression. A Mises-type criterion is thus not able to accurately predict the transformation surface in the 
stress space at a given temperature. It has been shown, using a micromechanical model, that the tension-compression asymmetry is linked to the loss of symmetry induced by the appearance of martensitic variants [3]. The tension-compression asymmetry is observable not only on the critical stress to trigger the martensitic transformation, but also on the stress-temperature slopes of the phase diagram and on the magnitude of transformation strains [4]. The behavior of processed SMAs also shows a strong anisotropic behavior [5, 6]. Like other metals, the texture of the material and the presence of internal stresses are found to be responsible for such anisotropy. A micromechanical analysis has shown that the transformation of a textured polycrystal is strongly anisotropic [7]. Recent efforts have focused on the development of suitable criteria to take into account the anisotropic behavior of precessed SMAs. It is shown that the transformation surfaces could be well predicted, but the evolution of transformation strains should be investigated even further [8].

Nowadays, the design strategy for identifying the parameters of anisotropy relies more and more on numerical simulations using Finite Element Analysis (FEA). An efficient analysis tool thus should be based on a macroscopic continuum model implemented in FEA software that can integrate comprehensive transformation criteria which describe the aforementioned effects. Predicting the start and finish of the transformation procedure with as much precision as possible is also of outmost importance, since the strains produced during transformation are large in scale and an inaccurate prediction can overthrow the validity of a numerical simulation. The introduction of the effect of processing conditions, i.e., anisotropic transformation behavior, is important as well, since most of the SMA structures are made out of wires or plates. The effect of drawn and rolled processing conditions has to be taken properly into account to obtain an efficient, accurate design tool.

Some established models make use of the Von Mises criterion to define the constitutive behavior of SMAs [913]. This choice is primarily motivated by the small number of parameters to be identified and the specific use of the structures to be designed (e.g., linear actuators). Constitutive models that include tension-compression asymmetry have been developed in the last 20 years $[1,14-$ 17]. They rely on a transformation criterion that depends on the invariants of the stress tensor. Following the normality rule for the transformation strains, associativity to such criterion is characterized by the invariance of transformation power with respect to loading direction [18]. At the point of saturation, an analytical expression of the transformation strains can thus be expressed. This expression can therefore be utilized as a bound for evolution of transformation strain [14]. The transformation surface in the stress space can then be derived starting from these physical bounds of the transformation strain. The criterion introduced by Taillard et al. [7] is able to reproduce the anisotropic transformation, but does not focus on the definition of an evolution equation that could accurately predict the direction of transformation. As for the shape of the transformation surface itself, it appears that quadratic anisotropic yield criteria which are commonly used for a wide variety of materials in numerical computations, such as Hill [19] or its more general form, Tsai-Wu [20] do not fit well the data obtained by experiments. In the mentioned models [7, 18, 20], the particular pear shape of the Prager equation introduced for SMAs by Patoor et al. [3] seems to fit better the experimental observations.

In this work, an effort to produce a new suitable transformation criterion for loading of SMAs under a constant temperature is presented. The purpose is to introduce a criterion that uses the components of the stress tensor and is independent of the transformation strain tensor to form a surface in the space of stresses which determines when transformation in SMAs starts and how it evolves. This criterion proves successful in expressing both asymmetry and anisotropy and also captures multiple results that might come either from experiments or from micromechanical simulations. The second main purpose of the work is to develop a formulation that can predict the evolution of transformation strains for textured materials.

A micromechanical model has previously been developed based on the reference work of Patoor et al. [21]. The input to the micromechanical model is crystallographic orientation of the grains that form the SMA polycrystal. The expected outcome from a polycrystal with random orientation is isotropic yield surface and inelastic strain evolution, whereas textured polycrystal would exhibit anisotropy in transformation [22, 23]. By conducting the micromechanics calculations of textured material and then calibrating the proposed equations, the aim is to come up with a consistent scheme which enables the direct connection between the processing conditions and the macroscopic effect on anisotropy that can be implemented in FEA packages for structural design.

The present paper is organized as follows: in the first part of this study, the mathematical configuration of the new criterion is presented. The convexity and the connection of the formulation with other criteria are investigated. In the second part, two ways of achieving the transition to the space of strains are presented and discussed. The third part is dedicated to the evaluation of the new criterion using results acquired from the micromechanical model and to a discussion about the capability of the present development to capture texture effects.

In the terminology of this paper, experimental results refer either to actual results from experiments, or to results 
from simulations obtained through the micromechanical model, or both. Furthermore, the following notations are used:

\section{$\alpha: \quad$ representing a scalar. \\ $\alpha$ : representing a second- or fourth-order tensor. \\ $\alpha^{\prime}$ : $\quad$ representing the deviatoric part of a second- order tensor. \\ $\operatorname{tr}(\mathbf{A}): \quad$ the trace of a second-order tensor denoted $\mathbf{A}$. \\ $\operatorname{Det}(\mathbf{A})$ : representing the determinant of a second-order tensor or a matrix denoted $\mathbf{A}$. \\ A : B: representing the double contraction product of the tensor $\mathbf{A}$ to the tensor $\mathbf{B}$. \\ New Transformation Criterion and Evolution Equation for Transformation Strain}

For the purpose of giving a context to the proposed approach of modeling the particular aspect of anisotropy in transformation, it is essential to describe beforehand the general framework of thermodynamics in which this work is developed.

Generally, the macroscopic behavior of SMAs is approached by means of using suitable constitutive equations which involve state variables of the material [13]. Thermodynamic state variables are those that represent all quantities that characterize a material body at a certain state [24]. If they can be observed, they are called external state variables, otherwise internal state variables [25]. Henceforth, the set of all the internal variables will be denoted as $\mathbf{V}$.

Those constitutive equations are derived through a prescribed thermodynamic potential. This is a function that characterizes a certain thermodynamic state of the body and depends on the state variables. At every state, the thermodynamic potential represents a quantity of energy within the material system. Therefore, it evokes products of the state variables with their thermodynamically conjugant quantities, called the general thermodynamic forces (GTFs). The set of all GTFs will be henceforth denoted as A.

It is commonly shown that the transformation strain is thermodynamically conjugant to stress, usually by implementing the procedure first applied by Coleman and Noll [26] under the conditions described by Lubliner [27]. All the basic laws of continuum mechanics need to be validated through the implementation of the thermodynamic potential, including the second law of thermodynamics, usually expressed by the local form of the Clausius-Duhem inequality [28].

SMAs are considered to behave in an elastic manner when transformation does not occur and in a rate- independent, non-linear manner only when transformation occurs. For this reason, the existence of a thermoelastic domain is commonly accepted [17]. It is a closed mathematical hypersurface in the space of selected GTFs, for which the set of GTFs at any given state may only position the material within or on its bounds. In mathematical terms, it is given by

$\Phi(\mathbf{A}) \leqslant 0 \quad$ for all possible sets of $\mathbf{A}$.

When the state of the material lies within the bounds of the thermoelastic region, it behaves elastically and no dissipation is produced. When the state of the material lies on the bounds of the region, the rates $\dot{V}$ of the internal state variables produce dissipation that must comply with the Clausius-Duhem inequality. As a result, the thermoelastic domain is used as the sole criterion to determine whether the material undergoes transformation or not. It is therefore called a transformation criterion, and it is comparable to the yield criterion of plasticity for the case of martensitic transformation. It is found as

$\Phi(\mathbf{A})=0$.

The principle of maximum dissipation has been implemented in some successful models [12]. It assumes that the transformation that the SMA undergoes is characterized by maximum dissipation. This means that, out of all the admissible sets of rates of the internal variables, the one which leads to the maximum possible dissipation actually occurs. Simo and Hughes [29] employed this principle for the case of plasticity to demonstrate the convexity of the yield criterion in stress-internal state variable space, the normality and associativity of the internal variable evolution rules, and the presence of Kuhn-Tucker loading conditions. As a direct result of the implementation of the principle, the evolution rule under transformation of all internal state variables is determined by associativity as such:

$$
\dot{\mathbf{v}}=\dot{\lambda} \frac{\partial \Phi}{\partial \mathbf{A}}, \dot{\lambda}>0
$$

Viewing the transformation strain $\varepsilon^{t}$ as an internal variable, having established that the stress $\sigma$ is its GTF, it is concluded that

$\dot{\boldsymbol{\varepsilon}}^{t}=\dot{\lambda} \frac{\partial \Phi}{\partial \boldsymbol{\sigma}}$

Similarly, this time avoiding associativity, a transformation potential $Z(\boldsymbol{A})$ can be defined, from which $\dot{\boldsymbol{\varepsilon}}^{t}$ is considered to be derived:

$\dot{\boldsymbol{\varepsilon}}^{t}=\dot{\lambda} \frac{\partial Z}{\partial \boldsymbol{\sigma}}$

The notion of the dissipation potential for the case of plasticity is well described in [28]. The difference between 
(4) and (5) lies only in the selection of the dissipation potential. In both these equations, the variable $\dot{\lambda}$ is called the transformation multiplier, in equivalence with the plastic multiplier, as found in plasticity.

In the following sections of the current work, it will be examined if any of those conditions can hold true for the case of a criterion which is anisotropic in the space of stresses and for the case of a dissipation potential which is isotropic, respectively. When this relation fails to capture macroscopic observations, then a new evolution rule should be proposed and the whole principle of maximum dissipation does not hold [28].

\section{Introduction of the Altered Prager Criterion}

Patoor et al. [3] made use of the Prager equation to fit isotropic asymmetric results obtained from their self-consistent micromechanical model. The original form is

$h(\boldsymbol{\sigma})=\sqrt{J_{2}}\left(1+b \frac{J_{3}}{J_{2}^{3 / 2}}\right)^{\frac{1}{2}}-k=0$,

where $J_{2}$ and $J_{3}$ denote the second and third invariant of the deviatoric part of the stress tensor, respectively:

$$
J_{2}=\frac{1}{2} \sigma_{i j}^{\prime} \sigma_{i j}^{\prime}
$$

and

$J_{3}=\frac{1}{3} \sigma_{i j}^{\prime} \sigma_{j k}^{\prime} \sigma_{k i}^{\prime}$,

using the Einstein summation for double indices.

In this form (6), b corresponds to the actual representation of asymmetry. If $b=0$, the Prager equation reduces to the classical Von Mises criterion. The value $\mathrm{k}$ is the maximum shear stress under pure shear loading, exactly like Von Mises as well. The Prager equation is a valid yield criterion that expresses a convex surface for a specific range of b. Furthermore, it succeeds in expressing an isochoric plastic, or transformation in the case of SMAs, evolution rule, since $\operatorname{tr}\left(\frac{\partial h}{\partial \boldsymbol{\sigma}}\right)=0$.

In this paper, the notion of the alteration of the axes of the stress space is used, as in the work of Karafillis and Boyce [30] to retain the shape of the original transformation surface, but at the same time include anisotropy. On the other hand, the expression is extended to a more general form using a power function. Thus

$\hat{\Phi}_{\sigma}(\boldsymbol{\sigma})=\sqrt{J_{2}\left(\boldsymbol{\sigma}^{*}\right)}\left[1+b \frac{J_{3}\left(\boldsymbol{\sigma}^{*}\right)}{J_{2}^{3 / 2}\left(\boldsymbol{\sigma}^{*}\right)}\right]^{\frac{1}{n}}-k_{\sigma}=0$,

where $n$ is a positive real number,
$\boldsymbol{\sigma}^{*}=\boldsymbol{R}_{\boldsymbol{\sigma}}: \boldsymbol{\sigma}-\boldsymbol{x}_{\boldsymbol{\sigma}}$

$\boldsymbol{R}_{\boldsymbol{\sigma}}$ is a fourth-order dimensionless tensor which only contains constants and $\boldsymbol{x}_{\boldsymbol{\sigma}}$ is a second-order tensor with dimensions of stress.

In that way, a new space of stresses $\sigma^{*}$ is created, one that represents a distortion of the space of real stresses and is based on the nature of the tensor $\boldsymbol{R}_{\boldsymbol{\sigma}}$, the description of which is given below:

1. If the effects that $\boldsymbol{R}_{\boldsymbol{\sigma}}$ induces on the shear stresses are ignored and a three-dimensional vector $\sigma$ consisting of only the normal stresses is considered, then $\boldsymbol{R}_{\boldsymbol{\sigma}}$ reduces to a matrix expressed as $\boldsymbol{R}$ with the following properties:

(a) $\boldsymbol{R}$ is expressed as a product of three rotation matrices:

$$
\boldsymbol{R}=\boldsymbol{R}_{z} \boldsymbol{R}_{x} \boldsymbol{R}_{p}
$$

Each of these three matrices represents a simple rotation of the space of normal stresses $\sigma_{11}$ $\sigma_{22}-\sigma_{33}$ and can be formed out of a single value representing the respective angle: $\boldsymbol{R}_{\boldsymbol{z}}$ represents a rotation around the $\sigma_{33}$ axis by an angle $\theta_{z} . \boldsymbol{R}_{\boldsymbol{x}}$ represents a rotation around the $\sigma_{11}$ axis by an angle $\theta_{x} . \boldsymbol{R}_{\boldsymbol{p}}$ represents a rotation around the $\sigma_{11}=\sigma_{22}=\sigma_{33}$ axis by an angle $\theta_{p}$.

(b) The result of this expression makes $\boldsymbol{R}$ a rotation matrix itself: it is orthogonal and $\operatorname{Det}(\boldsymbol{R})=1$.

2. Extending $\boldsymbol{R}$ in order to achieve a transformation of the six-dimensional space of stresses including the shears results in the fourth-order $\boldsymbol{R}_{\boldsymbol{\sigma}}$.

Instead of extending the notion of rotation to six dimensions, the transformation of shears is expressed in a simpler way, which is the homogeneous anisotropic scaling of the respective axes: in the basic configuration (7), $\hat{\Phi}_{\sigma}$ is expressed by simple scaled functions of the shear stresses:

$\sigma_{i j}^{*}=l_{i j} \sigma_{i j}$ for $\mathrm{i} \neq \mathrm{j}$ where $\mathrm{l}_{\mathrm{ij}}=\mathrm{l}_{\mathrm{ji}}>0$.

The Einstein summation is not used here.

Eventually, $\boldsymbol{R}_{\boldsymbol{\sigma}}$ introduces the operation of distortion rather than rotation. More details about the formulation of $\boldsymbol{R}_{\boldsymbol{\sigma}}$ and its sub-components are found in Appendix 1 .

On the other hand, $\boldsymbol{x}_{\boldsymbol{\sigma}}$ represents a translation of the origin of axes by the constant vector $\left\{\sigma_{o 1}, \sigma_{o 2}, 0\right\}$. The analytical expression of the six components of the transformed stress space is finally as follows:

$$
\left\{\sigma_{11}^{*}, \sigma_{22}^{*}, \sigma_{33}^{*}\right\}^{\mathrm{T}}=\boldsymbol{R}_{\boldsymbol{z}} \boldsymbol{R}_{\boldsymbol{x}} \boldsymbol{R}_{\boldsymbol{p}}\left\{\sigma_{11}, \sigma_{22}, \sigma_{33}\right\}^{\mathrm{T}}-\left\{\sigma_{o 1}, \sigma_{o 2}, 0\right\} \text {. }
$$

It is worth noting that the operation 
$\boldsymbol{R}_{\sigma}: \boldsymbol{\sigma}$

is not equivalent to a rotation of the system coordinates, as in

$\sigma^{*}=\boldsymbol{R} \sigma \boldsymbol{R}^{T}-\boldsymbol{x}_{\sigma}$

except if $\boldsymbol{R}$ is the fourth-order identity tensor. However in that case, the isotropic formulation is reached again. Since the criterion aimed to be utilized depends only on the invariants of the stress deviator, it would be useless to change merely the coordinates, because the value of the invariants would remain the same. The choice of introducing rotations around the $\sigma_{11}$ and $\sigma_{33}$ axes is purely arbitrary. The case is the same for the choice of introducing the translation of the axes along the direction of the $\sigma_{11}$ and $\sigma_{22}$ axes. One could consider rotations and translations with respect to any choice of axes, as long as they induce linearly independent transformations.

One important difference observed between the original and the altered Prager surface is that $b$ is not directly connected to asymmetry. Since the altered Prager equation (7) introduces 9 completely independent values $\left\{\theta_{p}, \theta_{x}, \theta_{z}, \sigma_{o 1}, \sigma_{o 2}, k_{\sigma}, l_{12}, l_{23}, l_{13}\right\}$ which in turn induce 9 independent transformations, it could capture up to 9 points in the stress space for a choice of $b$ in a wide range. The parameter $b$ is still the main component which controls the shape of the surface.

\section{Convexity Analysis and Connection with Other Criteria}

When attempting to conduct a convexity analysis, we begin by considering convexity only in the $\sigma_{11}-\sigma_{22}-\sigma_{33}$ space. The shape of the surface in the space of normal stresses is a rotation of the surface described by

$\hat{\Phi}_{\sigma}^{o}(\boldsymbol{\sigma})=\sqrt{J_{2}(\boldsymbol{\sigma})}\left[1+b \frac{J_{3}(\boldsymbol{\sigma})}{J_{2}^{3 / 2}(\boldsymbol{\sigma})}\right]^{\frac{1}{n}}-k_{\sigma}=0$.

Therefore, $\hat{\Phi}_{\sigma}(\boldsymbol{\sigma})=0$ is convex as long as $\hat{\Phi}_{\sigma}^{o}(\boldsymbol{\sigma})=0$ is convex. This realization makes the convexity analysis much simpler. Here, the convexity analysis is straightforward and utilizes simple theorems regarding convexity of surfaces described by an equation of a form such as $\hat{\Phi}_{\sigma}^{o}(\boldsymbol{\sigma})=0$ : the Hessian

$H\left(\hat{\Phi}_{\sigma}^{o}\right)=\nabla^{2}\left(\hat{\Phi}_{\sigma}^{o}(\boldsymbol{\sigma})\right)$

is found in the $\sigma_{11}-\sigma_{22}-\sigma_{33}$ space. It is a $3 \times 3$ symmetric matrix. The convexity of the surface is guaranteed when $\mathrm{H}$ is positive definite, that is when its three leading principal minors are positive for all $\sigma_{11}, \sigma_{22}, \sigma_{33}$. The procedure follows well-established methods described in
[31, 32]. This is a two-way deduction: if the surface is convex, then $\mathrm{H}$ must be positive definite. The ending result is a range of values that $\mathrm{b}$ can take. For whichever set of $\left\{\sigma_{11}, \sigma_{22}, \sigma_{33}\right\}, \mathrm{H}$ is positive definite as long as $\mathrm{b}$ lies within this range. Therefore, the following statement can be made:

$$
\left(\hat{\Phi}_{\sigma} \text { and } \hat{\Phi}_{\sigma}^{o} \text { are convex }\right) \Leftrightarrow|b|<\frac{n \sqrt{27}}{2(9-n)} \leqslant \frac{\sqrt{27}}{2} \quad \text { for } \mathrm{n}<4.5 \text {. }
$$

One conclusion of this is a profound effect for the original isotropic Prager surface (6), since b is the physical representation of asymmetry. Indeed, it can be written in the following form: $b=\left(\frac{\gamma-1}{\gamma+1}\right) a$, where $a=\frac{3 \sqrt{3}}{2} \approx 2.598$ and $\gamma=\left(\frac{\sigma_{\text {comp }}}{\sigma_{\text {trac }}}\right)^{n}$.

Here, $\sigma_{\text {comp }}$ and $\sigma_{\text {trac }}$ denote the stress level for the start of transformation in unidirectional compression and tension, respectively. The limitation of $b$ induces a limitation of the ratio $\gamma$ as well, in order to ensure convexity: $\gamma^{\frac{1}{n}}=\frac{\sigma_{\text {comp }}}{\sigma_{\text {trac }}}<\left(\frac{9}{9-2 n}\right)^{\frac{1}{n}}$.

This means that the isotropic Prager equation would fail to describe the yield or transformation surface of a material for which the scale of asymmetry exceeds this ratio.

This is not the case for the altered Prager criterion (7). It is noted that $\mathrm{b}$ could be considered an optimization factor and can take arbitrary values, and therefore it can always lie within the range that ensures convexity.

As for expanding the convexity analysis to the six-dimensional space which also includes the shear stresses, it proves to be a minor issue to resolve, since $\hat{\Phi}_{\sigma}(\boldsymbol{\sigma})$ is expressed as a function of a polynomial which is the sum of $l_{i j}^{2} \sigma_{i j}^{2}$ components when $i \neq j$. Therefore, convexity ensues.

One may also note how the proposed criterion can reduce to simpler forms. Firstly, considering $\boldsymbol{x}_{\boldsymbol{\sigma}}=0, \boldsymbol{R}_{\boldsymbol{\sigma}}=$ $\boldsymbol{I}$ (the fourth-order identity tensor), and $n=2$, the original isotropic Prager equation is yielded. On the other hand, if $\boldsymbol{R}_{\boldsymbol{\sigma}}$ still represents the said distortion of the stress space, but considering $n \rightarrow \infty$, it is shown that the equation is equivalent to a specific case of the Tsai-Wu criterion: indeed, $\frac{1}{n} \rightarrow 0$ and the effect of $J_{3}$ vanishes. Lastly, when $\boldsymbol{R}_{\boldsymbol{\sigma}}=\boldsymbol{I}, \boldsymbol{x}_{\boldsymbol{\sigma}}=0$, and $n \rightarrow \infty$, the simple Von Mises criterion is reached again.

\section{Capturing Anisotropy}

In the same manner that common anisotropic criteria such as Hill and Tsai-Wu make use of multiple coefficients (6 in 
the case of Hill's criterion, up to 36 for Tsai-Wu), there is a need to incorporate similar coefficients to simulate the anisotropic behavior under transformation for SMAs as well: on one hand, the asymmetric isotropic criterion of Prager does not succeed in describing anisotropy. On the other hand, even Tsai-Wu's criterion, the most general quadratic equation, does not capture the particular shape of the transformation surface appearing for SMAs. The manipulation of the parameters involved in the Tsai-Wu equation cannot change the ellipsoidal shape of the resulting surface, which is still away from experimental observations. On the contrary, the expression (5) succeeds in capturing nine experimental results from simple tension, compression, and shear tests and can form a criterion which can be used to describe the start of the forward transformation and its evolution. Six of those results must come from data regarding tests purely in normal stresses, and the remaining three must represent results in pure shear tests. The coefficients involved in the new criterion are to be considered material constants and calibrated through the experimental results. Actual experiments would require meticulous experimental techniques [33-35]. For the analysis of the capability of the developed criteria to take into account the specific feature of martensitic transformation in textured polycrystal, simulated experiments through a micromechanical model are preferred. This methodology has been proven accurate in capturing the multiaxial behavior of SMAs [7]. When trying to calibrate the proposed criterion to micromechanical results, two independent systems of non-linear equations are generated:

1. A $6 \times 6$ system where the target values are $\left\{\theta_{p}, \theta_{x}, \theta_{z}, \sigma_{o 1}, \sigma_{o 2}, k_{\sigma}\right\}$.

2. A $3 \times 3$ system where the target values are $\left\{l_{12}, l_{23}, l_{13}\right\}$. This system is further reduced to three independent systems with one target value for each.

The value of $b$ can still be considered independent. Therefore, $b$ can be treated as an optimization factor when trying to fit even more experimental results. However, if an abundance of data is available at hand that is more than 6 points available for the calibration of the surface in the $\sigma_{11}-\sigma_{22}-\sigma_{33}$ space, then the coefficient b can be considered just another target value to be determined. This can be achieved through an identification procedure that uses optimization algorithms. The error between the resulting surface and the set of data is eventually minimized.

Extending the concept of using an optimization procedure, one could also consider another transformation rule for the stress tensor, in the way of taking $\boldsymbol{x}_{\boldsymbol{\sigma}}=0$. That results to a simpler expression of the distorted stress space, one that does not include any translation of the origin of axes. On the other hand, the nature of the expressions used has a disadvantageous consequence: the mathematical problem at hand is highly non-linear and so far it is addressed using only numerical methods. The ending result is a finite yet large set of real solutions that describe different surfaces which all capture the experimental data. The optimal solution to choose from that set is a matter of best fit with the rest of the experimental data. Rigorous optimization techniques need to be implemented, such as the ones used in works regarding parameter identification [36].

\section{Evolution Equations of Transformation Strain}

Several constitutive models make use of a scalar $\varepsilon_{e q}^{t}$ called the equivalent transformation strain [14, 37], which is a function of $\varepsilon^{t}$, to describe at which point the forward transformation is found. In several models [7, 18, 38], $\varepsilon_{e q}^{t}$ is considered to be in direct relation with the martensitic volume fraction (MVF) $\xi$.

The relation between the rates of stress and transformation strain is called the evolution equation of transformation strain, viewing the strain as the GTF of stress. The most general form of this relation is identical to (5), choosing a transformation potential $\hat{Z}(\boldsymbol{\sigma})$. Following a direct relation with $\xi$, a more specific equation is found:

$\dot{\boldsymbol{\varepsilon}}^{t}=\dot{\xi} \frac{\partial Z}{\partial \boldsymbol{\sigma}}$.

When $\hat{Z}(\boldsymbol{\sigma})$ is chosen to be the transformation surface, the maximum dissipation principle is satisfied [17] and

$\dot{\boldsymbol{\varepsilon}}^{t}=\dot{\xi} \frac{\partial \Phi}{\partial \boldsymbol{\sigma}}$

In this work, it is examined how three particular evolution rules for the transformation strain behave with comparison to simulated experimental results. The first rule is consistent with (15) and the second with (14). In addition to that, a third evolution rule will be introduced, $\dot{\boldsymbol{\varepsilon}}_{r}$, and the three options given will be compared.

Firstly, we define

$\dot{\boldsymbol{\varepsilon}}_{a s}^{t}=\dot{\xi} H \frac{\partial \hat{\Phi}_{\sigma}}{\partial \boldsymbol{\sigma}}$.

This formulation is consistent with the maximum dissipation principle and expresses the rule of associativity. By comparing with (4), it is concluded that the transformation potential is indeed the transformation function. The rate of $\boldsymbol{\varepsilon}^{t}$ is directly linked to $\dot{\xi}$, which is a common practice in various existing works $[11,12]$. In a very recent model for anisotropic SMA behavior [39], an interesting evolution equation has been proposed, also based on the work of Lagoudas and co-workers. The variable $H$ is a scale factor and expresses the magnitude of the rate at which $\boldsymbol{\varepsilon}^{t}$ increases. Usually, it is a function of stresses: 
$H= \begin{cases}H_{\min } ; & \bar{\sigma} \leqslant \bar{\sigma}_{\text {crit }}, \\ H_{\min }+\left(H_{\text {sat }}-H_{\min }\right)\left(1-e^{-k\left(\bar{\sigma}-\bar{\sigma}_{\text {crit }}\right)}\right) ; & \bar{\sigma}>\bar{\sigma}_{\text {crit }},\end{cases}$

as taken directly from [40]. In the latter, $\bar{\sigma}=\sqrt{3 J_{2}}$ and $H_{\text {sat }}, H_{\text {min }}, k, \bar{\sigma}_{\text {crit }}$ are material parameters.

Secondly, we define

$\dot{\boldsymbol{\varepsilon}}_{\text {iso }}^{t}=\dot{\xi} H \frac{\partial \hat{\Phi}_{\sigma}^{o}}{\partial \boldsymbol{\sigma}}$

This formulation comes from the consideration of $\hat{\Phi}_{\sigma}^{o}$ found in (12) as the dissipation potential $Z$ found in (5). The derivatives $\frac{\partial \hat{\Phi}_{\sigma}^{o}}{\partial \boldsymbol{\sigma}}$ and $\frac{\partial \hat{\Phi}_{\sigma}}{\partial \boldsymbol{\sigma}}$ found in (17) and (16), respectively, express a tensor which is normal to the respective potentials. The first derivative is called $\boldsymbol{\Lambda}_{\boldsymbol{o}}$ and it takes the expression as follows:

$$
\begin{aligned}
\boldsymbol{\Lambda}_{\boldsymbol{o}}(\boldsymbol{\sigma})= & \frac{\partial \hat{\Phi}_{\sigma}^{o}(\boldsymbol{\sigma})}{\partial \boldsymbol{\sigma}}=\left(1+b \frac{J_{3}(\boldsymbol{\sigma})}{J_{2}^{3 / 2}(\boldsymbol{\sigma})}\right)^{\frac{1}{n}-1} \\
\times & {\left[\frac{\boldsymbol{\sigma}^{\prime}}{2 \sqrt{J_{2}(\boldsymbol{\sigma})}}+\frac{b}{6 n J_{2}^{2}(\boldsymbol{\sigma})}\left(6 J_{2}(\boldsymbol{\sigma}) \boldsymbol{\sigma}^{\prime} \cdot \boldsymbol{\sigma}^{\prime}-4 J_{2}^{2}(\boldsymbol{\sigma}) \boldsymbol{I}\right.\right.} \\
& \left.\left.+(3 n-9) J_{3}(\boldsymbol{\sigma}) \boldsymbol{\sigma}^{\prime}\right)\right]
\end{aligned}
$$

where $\boldsymbol{I}$ is the identity second-order tensor. On the other hand,

$\frac{\partial \hat{\Phi}_{\sigma}}{\partial \boldsymbol{\sigma}}=\frac{\partial \hat{\Phi}_{\sigma}^{o}\left(\boldsymbol{\sigma}^{*}\right)}{\partial \boldsymbol{\sigma}}=\frac{\partial \boldsymbol{\sigma}^{*}}{\partial \boldsymbol{\sigma}}: \frac{\partial \hat{\Phi}_{\sigma}^{o}\left(\boldsymbol{\sigma}^{*}\right)}{\partial \boldsymbol{\sigma}^{*}}$

according to the chain rule for derivation.

According to (8), it is considered $\frac{\partial \boldsymbol{\sigma}^{*}}{\partial \boldsymbol{\sigma}}=\boldsymbol{R}_{\boldsymbol{\sigma}}$ and in (19) it is found that

$$
\frac{\partial \hat{\Phi}_{\sigma}}{\partial \boldsymbol{\sigma}}=\boldsymbol{R}_{\boldsymbol{\sigma}}: \boldsymbol{\Lambda}_{\boldsymbol{o}}\left(\boldsymbol{\sigma}^{*}\right)
$$

It is noted that $\boldsymbol{\Lambda}_{\boldsymbol{o}}(\boldsymbol{\sigma})$ is a deviatoric tensor, as expected from deriving a scalar function of the invariants of the stress deviator.

Finally, we define

$\dot{\boldsymbol{\varepsilon}}_{\boldsymbol{r}}^{t}=\dot{\xi} H\left(\boldsymbol{r}_{\varepsilon} \cdot \boldsymbol{\Lambda}_{o} \cdot \boldsymbol{r}_{\varepsilon}^{T}\right)$

Here, a rotation of the normal $\boldsymbol{\Lambda}_{o}$ of $\dot{\boldsymbol{\varepsilon}}_{2}^{t}$ is introduced, thus rotating the whole tensor that comes from the expression (17). This rotation comes from an angle $a^{\varepsilon}$ lying on the plane given by $\varepsilon_{11}^{t}+\varepsilon_{22}^{t}+\varepsilon_{33}^{t}=0$. As with $\boldsymbol{R}_{\boldsymbol{p}}$, the rotation matrix $\boldsymbol{r}_{\varepsilon}$ is given by

$\boldsymbol{r}_{\varepsilon}=\left(\begin{array}{ccc}\cos a^{\varepsilon}+u^{\varepsilon} & u^{\varepsilon}-v^{\varepsilon} & u^{\varepsilon}+v^{\varepsilon} \\ u^{\varepsilon}+v^{\varepsilon} & \cos a^{\varepsilon}+u^{\varepsilon} & u^{\varepsilon}-v^{\varepsilon} \\ u^{\varepsilon}-v^{\varepsilon} & u^{\varepsilon}+v^{\varepsilon} & \cos a^{\varepsilon}+u^{\varepsilon}\end{array}\right)$ with $u^{\varepsilon}=\frac{\cos a^{\varepsilon}}{3}$ and $v^{\varepsilon}=\frac{\sin a^{\varepsilon}}{\sqrt{3}}$. However, unlike $\theta_{p}$ which is considered constant as found in $\boldsymbol{R}_{\boldsymbol{p}}$, the value of $a^{\varepsilon}$ is a function of $\boldsymbol{\Lambda}_{\boldsymbol{o}}$ :

$$
\begin{aligned}
a^{\varepsilon}= & a_{o}^{\varepsilon}+2 \sqrt{\frac{F^{\varepsilon}}{\pi}} \operatorname{Exp}\left[-F^{\varepsilon}\left(\left(\omega-\omega_{o}^{\varepsilon}\right)^{2}+\left(c^{\varepsilon}\right)^{2}\right)\right] \\
& \sinh \left[2 F^{\varepsilon} c^{\varepsilon}\left(\omega-\omega_{o}^{\varepsilon}\right)\right] .
\end{aligned}
$$

In the latter,

$$
\omega\left(\boldsymbol{\Lambda}_{o}: \boldsymbol{B}_{o}^{\varepsilon}\right)= \begin{cases}\arccos \left(\boldsymbol{\Lambda}_{o}: \boldsymbol{B}_{o}^{\varepsilon}\right) ; & \Lambda_{o 22} \geqslant 0 \\ 2 \pi-\arccos \left(\boldsymbol{\Lambda}_{\boldsymbol{o}}: \boldsymbol{B}_{\boldsymbol{o}}^{\varepsilon}\right) ; & \Lambda_{o 22}<0\end{cases}
$$

where $\Lambda_{o 22}$ is the second diagonal component of $\Lambda_{o}$.

The rest of the variables that appear other than $\Lambda_{o}$ are constants. The equation (23) introduces the rotation necessary to capture the effect of anisotropy to $\dot{\boldsymbol{\varepsilon}}^{t}$. It is considered that $a_{o}^{\varepsilon}=-\theta_{p}$, whereas $\boldsymbol{B}_{\varepsilon}^{\boldsymbol{o}}$ takes the following form:

$\boldsymbol{B}_{\varepsilon}^{\boldsymbol{o}}=\left(\begin{array}{ccc}2^{-1 / 2} & 0 & 0 \\ 0 & 0 & 0 \\ 0 & 0 & -2^{-1 / 2}\end{array}\right)$

The choice for the tensor of reference $\boldsymbol{B}_{\boldsymbol{\varepsilon}}^{\boldsymbol{o}}$ as the origin for measuring the angles is arbitrary. It represents the intersection of the $\varepsilon_{11}^{t}+\varepsilon_{22}^{t}+\varepsilon_{33}^{t}=0$ with the plane $\varepsilon_{22}^{t}=0$. The criterion $\Lambda_{o 22} \geqslant 0$ found in (24) is a direct result of this choice.

The variables $c^{\varepsilon}, F^{\varepsilon}, \omega_{o}^{\varepsilon}$ should be viewed as material constants and calibrated according to experimental results. The angles taken in mind for the rotation rule described by (21) and (23) are demonstrated in the schematic representation of Fig. 1. The vectors represent the normal components of the respective tensors and lie on the $\varepsilon_{11}^{t}+\varepsilon_{22}^{t}+\varepsilon_{33}^{t}=0$ plane.

Another remark for the evolution rule introduced in (21) is that it is not given as a direct derivative of a dissipation potential. A surface $\hat{Z}(\boldsymbol{\sigma})$, for which every $\dot{\boldsymbol{\varepsilon}}_{\boldsymbol{r}}^{t}$ is normal to, exists but its exact form is unknown.

\section{Numerical Simulations Based on the Micromechanical Model}

\section{Brief Description of the Micromechanical Model}

The numerical simulation of the constitutive response of polycrystalline SMAs is based on a micromechanical model developed by Patoor et al. [21], implementing the self-consistent scale transition method [41]. It is a method that has been proven robust and has been implemented in 


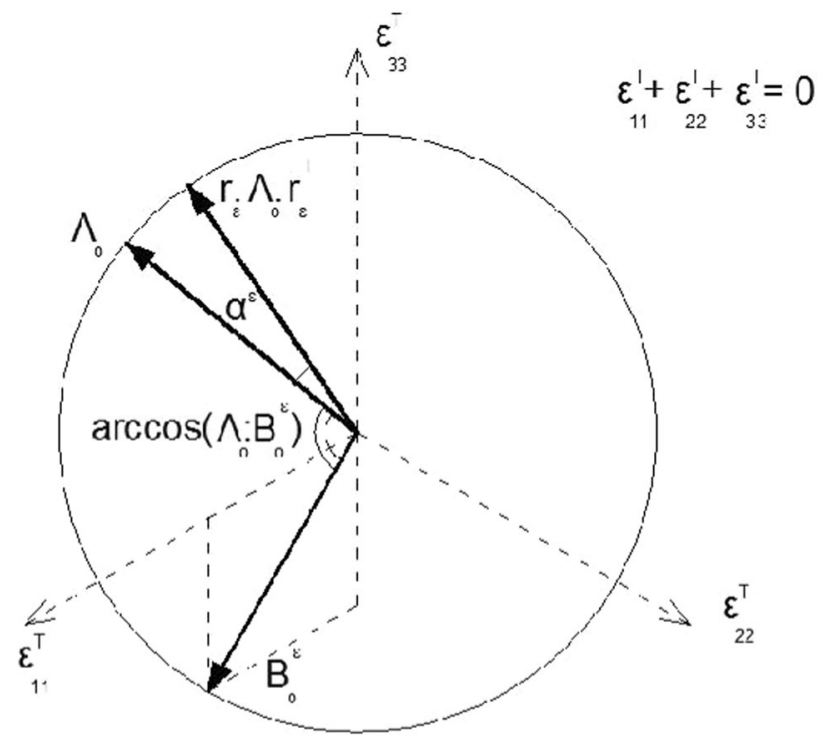

Fig. 1 The angles involved in the rotation of $\dot{\boldsymbol{\varepsilon}}_{r}^{t}$

other micromechanical models as well, e.g., in [42]. The model describes the local thermomechanical behavior inside a single grain from the crystallographic nature of the martensitic transformation and eventually obtains the overall, effective behavior of the polycrystal. At the local (grain) scale, the thermomechanical constitutive model is derived from a thermodynamical potential. The internal variables are defined as the volume fraction of each of the possible variants according to the crystallographic nature of the crystal. The transformation strain is defined from the Wechsler, Liebermannand Read theory, according to the direction of the normal to the habit plane [43, 44]. The evolution of the volume fraction of each of the variant systems is defined from its associated thermodynamic force [3]. A criterion for forward and reverse transformation is thus defined, and an adaptive Newton-Raphson algorithm is utilized to determine the volume fraction of each of the variant system accordingly. This operation is conducted for all the grains of the polycrystal at each time increment.

Inside a single grain, the martensitic transformation is mainly based on the following assumptions: The lattice vectors of austenite and martensite possess mutual orientation relationships that depend on the crystallographic nature of the alloy [45]. Martensitic transformation produces predominantly a shear strain along well-defined planes, which is crystallographically reversible [46].

It is worth mentioning that the choice of this particular micromechanical model was made because it has already been used for the same purpose of assessing other macroscopic models [7]. Its value is furthermore proven by the influence that it has on other micromechanical models [47]. The thermodynamics involved in the microscopic scale have also influenced the formulation of macroscopic models $[14,48]$ and their application to structural design [49].

The data inputs to this model are the crystallographic texture, the transformation temperatures, the material coefficients identified from the state diagram of the SMA, the normal of habit planes, and the transformation directions of the 24 variants. An interaction matrix which defines at least two types of interaction (i.e., in the preset case, weak and strong) between the formed martensite variants in a grain is included, following [41]. All these parameters are physical. They do not depend on the past of the material, except for the crystallographic texture.

The crystallographic texture describing the different grain orientations permits to take into account the multiaxial behavior of the polycrystal related to the forming process [50]. It is well known that the initial crystallographic texture is an important parameter in the behavior of SMA [51, 52]. The use of the micromechanical model permits to follow the microstructure evolution during loading [38].

\section{Constitutive Equations of the Polycrystalline Model}

Following these assumptions, the evolution equation for the inelastic strain linked with the martensitic transformation $\left(\dot{\boldsymbol{\varepsilon}}^{t}\right)$ is written as follows:

$\dot{\boldsymbol{\varepsilon}}^{t}=\sum_{k} \dot{\xi}_{k} \boldsymbol{\varepsilon}_{k}^{t}$

where $\xi_{k}$ is the volume fraction of the k-th variant system and $\boldsymbol{\varepsilon}_{k}^{t}$ is the strain associated to such variant, according to the WLR theory [44]:

$\boldsymbol{\varepsilon}_{k}^{t}=g \boldsymbol{n} \otimes \boldsymbol{m}$.

The transformation strain associated to each variant is thus obtained through the knowledge of the shear transformation magnitude $g$, the unit vector normal to the invariant plane $\boldsymbol{n}$, and the vector pointing in the direction of the transformation $\boldsymbol{m}$ [38].

The evolution equation for the rate of martensite volume fraction is linked to the thermodynamical force associated to each variant system [3]. The interaction between variants is taken into account through an interaction matrix which differentiates two types of interactions (compatible and incompatible) between the formed martensite variants in a grain. The pair of compatible and incompatible variants are found using the Hill interfacial operators according to [43].The knowledge of the evolution of each volume fraction of the variant systems allows the computation of the average stress in the grain and the local tangent modulus, according to an imposed increment of total strain. This local increment of strain related to the r-th grain has to be compliant to the macroscopic boundary conditions, which is achieved using the incremental localization equation: 
Table 1 Loading paths for the polycrystal specimens

\begin{tabular}{lll}
\hline Label of simulation & Loading conditions & Relative angle to 1-1 axis \\
\hline 1 & Uniaxial tension in 1 & $0^{\circ}$ \\
2 & Biaxial tension in 1 and 2 & $45^{\circ}$ \\
3 & Uniaxial tension in 2 & $90^{\circ}$ \\
4 & Compression in 1-tension in 2 & $135^{\circ}$ \\
5 & Uniaxial compression in 1 & $180^{\circ}$ \\
6 & Biaxial compression in 1 and 2 & $225^{\circ}$ \\
7 & Uniaxial compression in 2 & $270^{\circ}$ \\
8 & Tension in 1-compression in 2 & $315^{\circ}$ \\
\hline
\end{tabular}

The non-uniaxial cases represent equibiaxial conditions
$\dot{\boldsymbol{\varepsilon}}^{r}=\boldsymbol{A}_{\boldsymbol{r}} \dot{\boldsymbol{E}}$,

where $\dot{\boldsymbol{\varepsilon}}^{r}$ is the rate of the local total strain tensor, $\dot{\boldsymbol{E}}$ is the rate of the macroscopic total strain and $\boldsymbol{A}_{\boldsymbol{r}}$ is the r-th localization tensor. According to the self-consistent scheme, the localization tensor is written as follows:

$\boldsymbol{A}_{\boldsymbol{r}}=\left[\boldsymbol{I}+\boldsymbol{S} \overline{\boldsymbol{M}}^{t}\left(\boldsymbol{L}_{\boldsymbol{r}}^{t}-\overline{\boldsymbol{L}}^{t}\right)\right]^{-1}$,

where $S$ is the respective Eshelby tensor [53], evaluated numerically based on the anisotropic effective modulus description [54]. The model is supplied with the orientation of each grain by means of Euler angles that can be obtained experimentally, e.g., by Electron BackScatter Diffraction (EBSD) technique. Every grain is therefore considered as one phase of the continuum. The anisotropic features are naturally induced by the texture of the provided grain set. Knowing the volume fraction $c_{r}$ of each grain, the effective stiffness $\overline{\boldsymbol{L}}^{t}$ (and its inverse, the effective compliance $\overline{\boldsymbol{M}}^{t}$ ) is computed from

$\overline{\boldsymbol{L}^{t}}=\sum_{r} c_{r} \boldsymbol{L}_{\boldsymbol{r}}^{\boldsymbol{t}} \boldsymbol{A}_{\boldsymbol{r}}$

\section{Data for the Polycrystalline Model}

The numerical simulations are computed using the microstructural parameters of a $\mathrm{Cu}-\mathrm{Zn}-\mathrm{Al}$ alloy, taken from [41]. These alloys show experimental results matching the simulations carried out by this micromechanical model. The transformation strain amplitude $g$ is 0.23 for all variants. The parameters of the interaction matrix are as follows: $1 \mathrm{MPa}$ describing the weak interaction and 260 MPa for the strong interaction. The elasticity is supposed to be isotropic and the same in the two phases.

In the next two parts, the micromechanical model is used for two purposes:

1. To acquire the necessary experimental results in order to calibrate the criteria. The model provides with the set of data used to calculate the target values of the equation (7) for the stress surface and (21) for the evolution of transformation strain.

2. To validate the shape and the accuracy of the transformation surfaces. The number of results acquired by the micromechanical model is higher than needed for calibration. Therefore, the remaining data can be used to evaluate how close the transformation surfaces are to the additional predictions of the micromechanical model.

All the results of that model are obtained after running the simulations of loading of three polycrystal specimens containing 1000 grains each in total. These polycrystals are considered to correspond to an isotropic, rolled, or drawn specimen, according to their texture. For the rolled specimen, the rolling direction was set to be along the axis 1-1. For the drawn specimen, the drawing direction was also set along the axis 1-1. Pole figures of these specimens can be found in Fig. 8 in Appendix 2. The results presented hereafter correspond to simulations following eight proportional loading paths assuming plane stress conditions with $\sigma_{33}=\sigma_{12}=0$ in stress-controlled loading. The loading paths are presented in Table 1. Four of those are uniaxial and the remaining four are biaxial in the directions of the $1-1$ and $2-2$ axes. In the biaxial cases, the magnitudes of the stress components $\sigma_{11}$ and $\sigma_{22}$ are kept equal.

\section{Assessment of the Transformation Function and Evolution Rules}

\section{Evaluation of the Proposed Transformation Function}

The first set of simulations represents proportional loading of a non-treated polycrystal. The resulting surfaces in the stress and the strain spaces were isotropic. Their shape is found in Figs. 2 and 4. The results were similar to those reported in [1] and [38]. 


\section{$\sigma 22$}

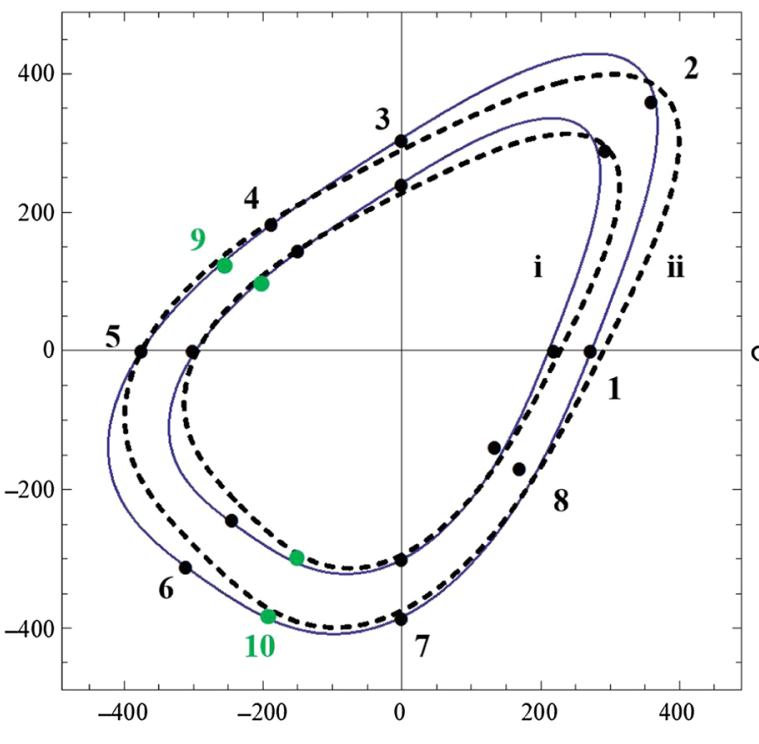

- Data -.. Isotropic surface — Anisotropic surface

Fig. 2 Evolution of transformation surface of rolled specimen for (i) $\xi=20 \%$ and (ii) $\xi=60 \%$ and comparison with isotropic surface

Table 2 Calibrated material parameters for rolled specimen

\begin{tabular}{ll}
\hline Identified parameter & Value \\
\hline$n$ & 2 \\
$b$ & 0.65 \\
$\theta_{p}$ & -0.0953 \\
$\theta_{x}$ & 0.0339 \\
$\theta_{z}$ & -0.0373 \\
$\sigma_{o 1}$ & $-15.17 \mathrm{MPa}$ \\
$\sigma_{o 2}$ & $-2.93 \mathrm{MPa}$ \\
$k_{\sigma}$ & $91.60 \mathrm{MPa}$ \\
\hline
\end{tabular}

The second set of simulations represents proportional loading of the rolled specimen. The procedure of matching the results of these simulations to fit the criterion described by (7) gives the set of target values, presented in Table 2 . Two more anisotropic surfaces were calibrated for a different assumption of the value of the factor $b$, in order to demonstrate the adaptability of the transformation criterion.

The third set of simulations represents proportional loading of the drawn specimen. Because of the higher level of anisotropy, b was selected here to be a target value and was calibrated at $b=0.59$.

Figures 2 and 4 show a comparison between all the results of the simulations and the criterion in terms of stresses obtained by (5), considering $n=2$. Good agreement is observed. Furthermore, it is shown in Fig. 3 that using alternative values for $b$ might result in better $\sigma 22$

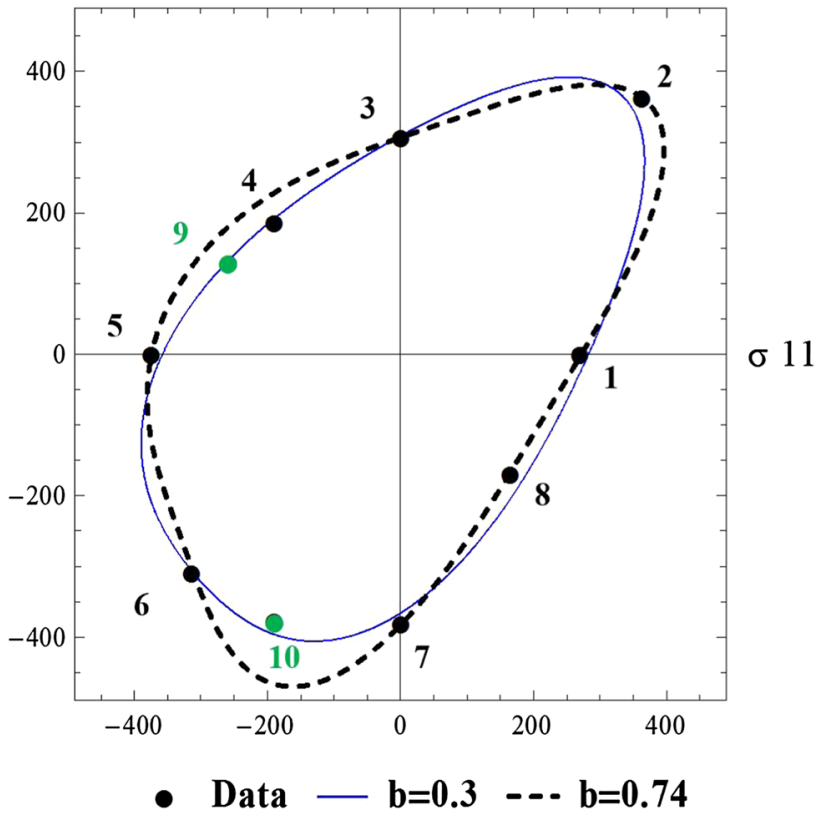

Fig. 3 Comparison between two anisotropic surfaces for different values of $\mathrm{b}$ for the rolled specimen for $\xi=60 \%$

surfaces, in the sense that they can capture better basic material properties, which here are the uniaxial strengths. Given an abundance of data, better calibration is induced by treating $\mathrm{b}$ as another target value. To that respect, two more loading paths are considered for the rolled specimen, labeled 9 and 10 in Figs. 2 and 3. It appears that the extreme in terms of convexity value $b=0.74$ is not as accurate as the values $b=0.65$ and $b=0.3$.

The evolution of the transformation surface as a function of $\xi$ also presents a very interesting effect. The detail of the transformation surfaces corresponding to two different MVFs in Fig. $4 b$ reveals that the sense of anisotropy may switch between directions as forward transformation progresses. Indeed, it is clear in Fig. 5 that the stress which corresponds to $1 \% \mathrm{MVF}$ for uniaxial tension in the 1-1 direction is higher than the stress for uniaxial tension in the 2-2 direction; but this is not the case when $\xi=10 \%$ : here, the stress is higher for tension along 2-2. The resulting surfaces accommodating these data are different in shape and not just in size. The size effect would be captured by a direct dependence of $\mathrm{k}$ on $\xi$, and this kind of evolution would be recognized as isotropic hardening [28]. However, in this case, more material parameters have changed between the two MVFs to capture the changing sense of anisotropy. Thus, an evolution of the material parameters is deemed necessary to capture the resulting stress-MVF curves. The proposed anisotropic surfaces in Fig. $4 \mathrm{~b}$ are calibrated separately according to data points for $\xi=1 \%$ 


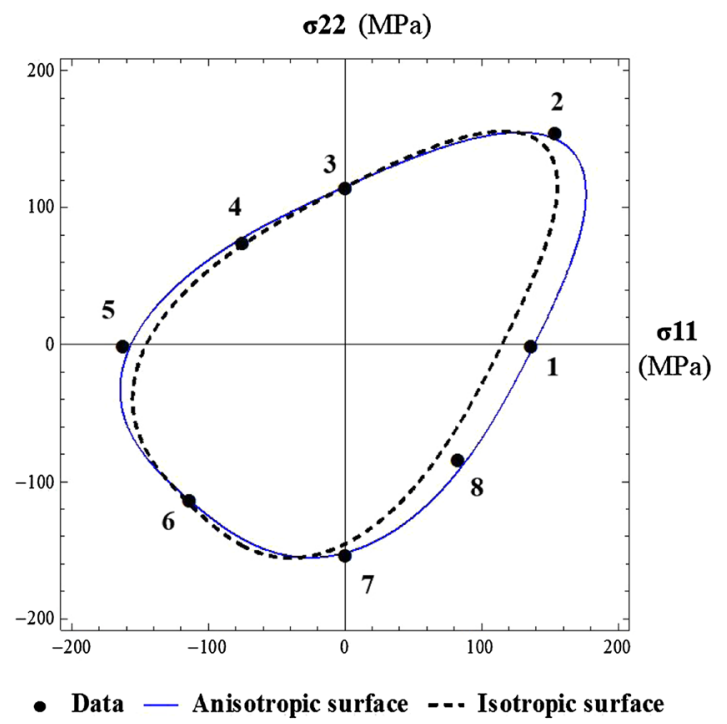

(a)

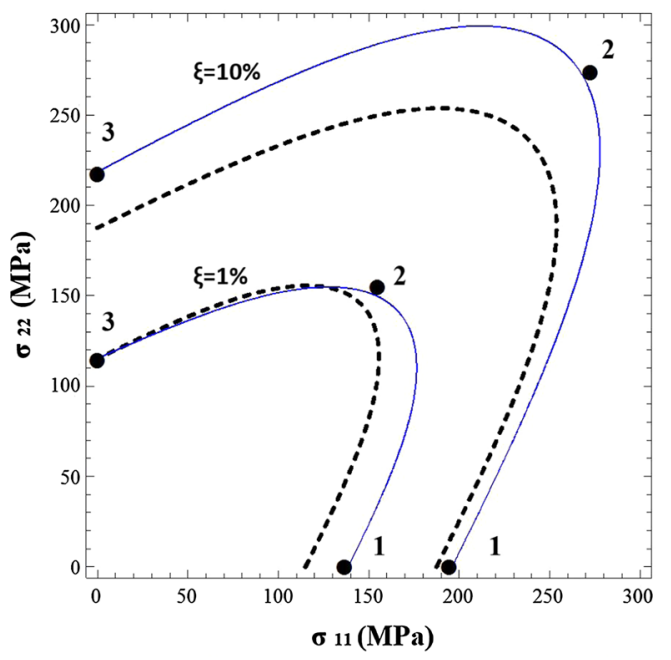

- Data — Anisotropic surface -..- Isotropic surface

(b)

Fig. 4 Comparison between isotropic and anisotropic surfaces for the drawn specimen for $\xi=1 \%$ (a) and evolution of transformation surface for $\xi=1 \%$ and $\xi=10 \%$ (b)

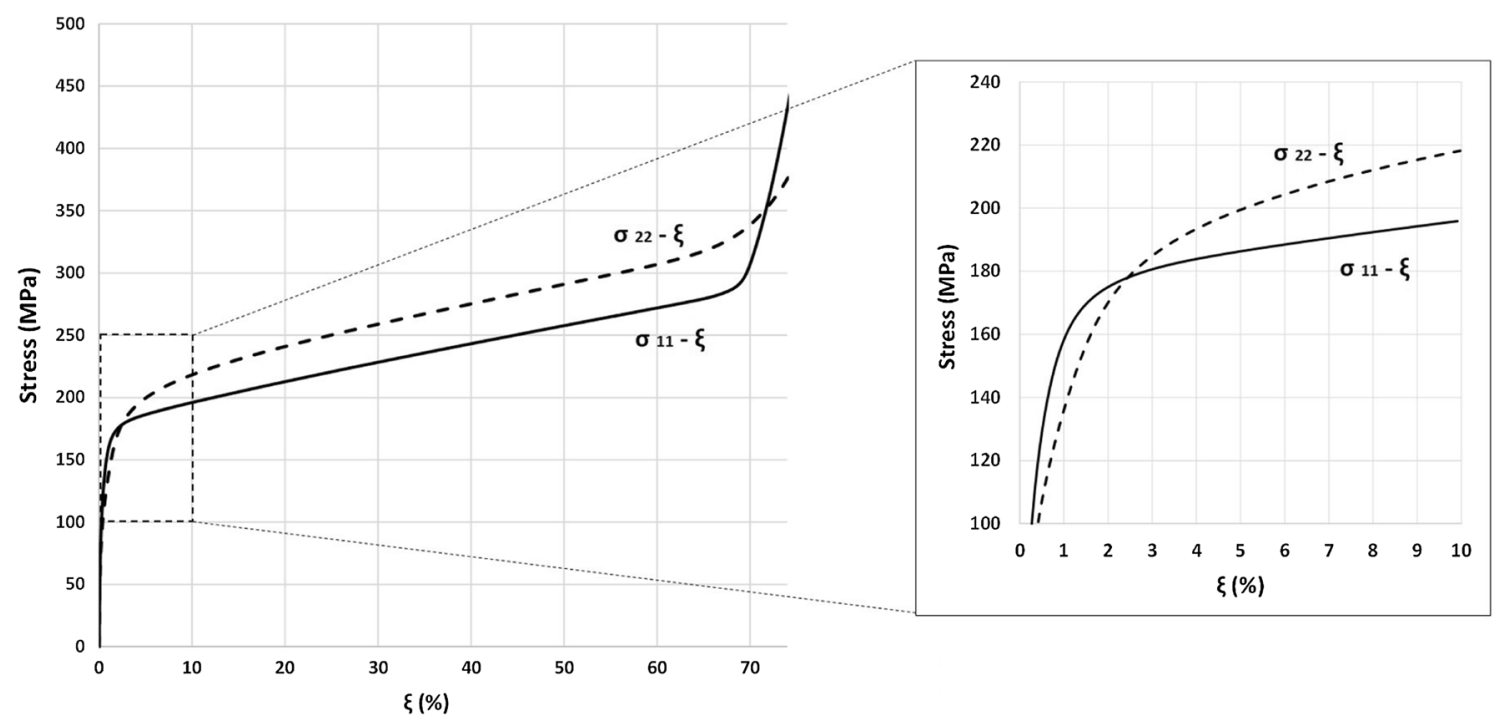

Fig. 5 Stress-MVF diagram for uniaxial tension in directions 1-1 and 2-2

and $\xi=10 \%$ with different sets of $\sigma_{o 1}, \sigma_{o 2}$, and $\mathrm{k}$. For the range of $\xi \in[5 \%, 70 \%]$, simple linear functions of $\sigma_{o 1}$, $\sigma_{o 2}$, and $\mathrm{k}$ could be considered that fit the evolution. However, more complex functions describing the hardening effect are needed for the implementation with a comprehensive thermodynamic model, such as the ones found in [25].

The importance of calibrating these surfaces extends to compensate for poor abilities to capture the compressioncompression strength in a real experiment because of buckling.
Evaluation of Evolution Equations for the transformation Strain

Using the evolution rules (16), (17), and (21), a large number of results regarding transformation strains were gathered. These results correspond to proportional loading under various directions. For every value of $\xi$, each evolution rule results in a respective locus on which the components of $\varepsilon^{t}$ lie. In the space of normal transformation strains, the three loci are flat shapes, meaning that they all lie on a plane. As demonstrated in Fig. 7, the loci 


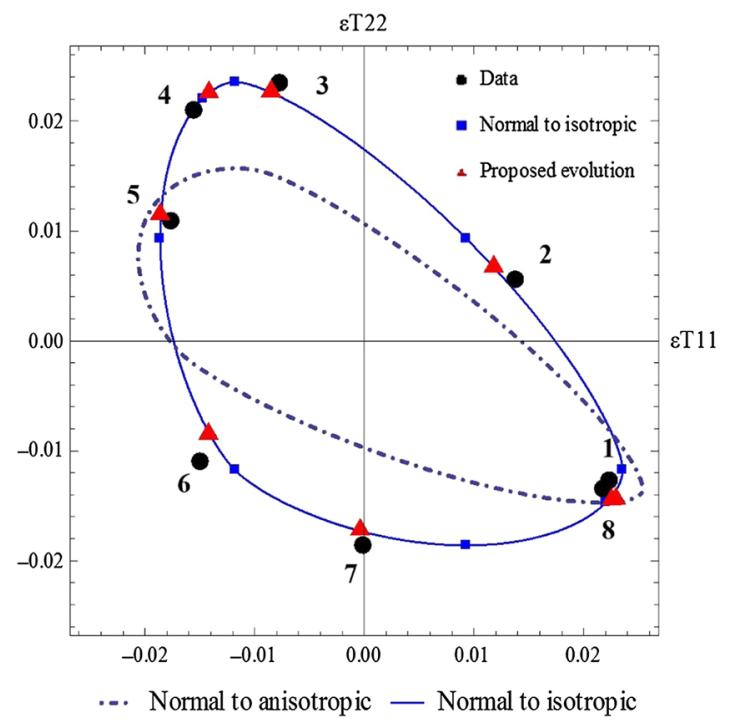

(a)

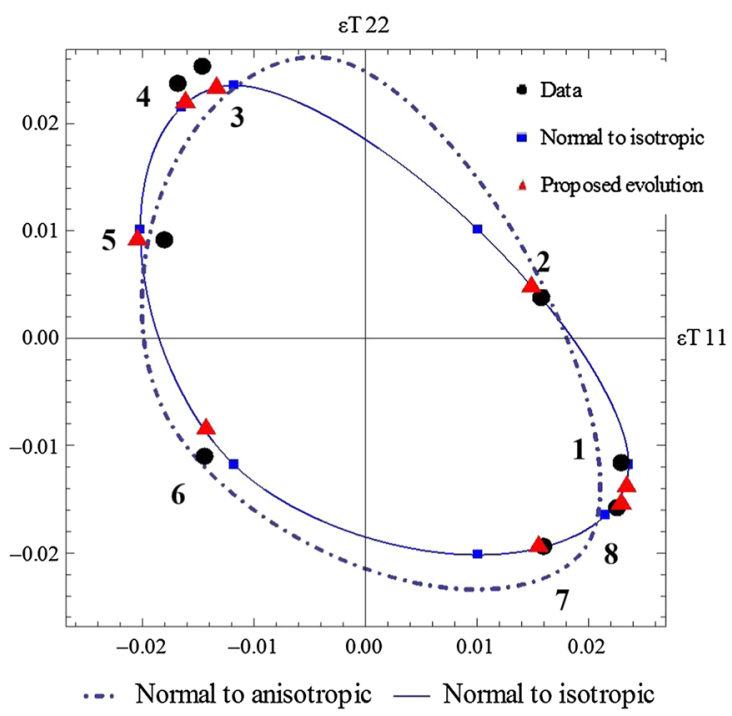

(b)

Fig. 6 Projection of the resulting transformation strains according to the proposed evolution law for the rolled (a) and the drawn specimen (b) for $\xi=70 \%$ on the plane $\varepsilon_{11}^{T}-\varepsilon_{22}^{T}$

$S_{i s o}$ and $\mathrm{S}_{\mathrm{r}}$ for the normal to the isotropic surface and the proposed evolution rules, respectively, coincide, whereas the locus $S_{a s}$ corresponding to the associative evolution equation lies on a different plane. The results for transformation strain which corresponds to $\xi=70 \%$ for the rolled specimen are also superposed to evaluate the accuracy of the flow rules. The loci $S_{i s o}$ and $\mathrm{S}_{\mathrm{r}}$ lie on the plane $\varepsilon_{11}^{t}+\varepsilon_{22}^{t}+\varepsilon_{33}^{t}=0$ : since $\dot{\varepsilon}_{i s o}^{t}$ has the same direction as the deviator $\Lambda_{\boldsymbol{o}}(\boldsymbol{\sigma})$, the resulting $\varepsilon_{2}^{t}$ must be deviatoric. On the other hand, since $\dot{\varepsilon}_{r}^{t}$ represents a rotation of $\dot{\varepsilon}_{i s o}^{t}$ around the $\varepsilon_{11}^{t}=\varepsilon_{22}^{t}=\varepsilon_{33}^{t}$ axis, $\dot{\varepsilon}_{r}^{t}$ is still a deviator and the resulting $\varepsilon_{r}^{t}$ is deviatoric as well. This means that the evolution rules (17) and (21) do succeed in representing transformation as an isochoric process, whereas (16) does not.

Even though the resulting loci $S_{i s o}$ and $\mathrm{S}_{\mathrm{r}}$ are identical, the respective evolution rules are not equivalent. While the shape on which they lie is common, the same loading direction corresponds to different positions on the shape. In Fig. $6 a, b$, only the strains resulting from the proposed criterion are accurate enough to be compared with data points. The results presented are a projection of the points shown in Fig. 7 on the $\varepsilon_{33}^{t}=0$. For every point on the surfaces corresponding to $\dot{\varepsilon}_{i s o}^{t}$ and $\dot{\varepsilon}_{r}^{t}$, it is $\varepsilon_{33}^{t}=-\varepsilon_{11}^{t}-\varepsilon_{22}^{t}$.

The fact that the resulting loci coincide for $\dot{\varepsilon}_{i s o}^{t}$ and $\dot{\varepsilon}_{r}^{t}$ is a direct consequence of the rotation imposed on the direction of $\dot{\varepsilon}_{i s o}^{t}$. This operation simply rearranges the points on the locus: every point is thus reassigned to different loading paths. This is clearly visible in Fig. 6a: all the points calculated through the proposed evolution

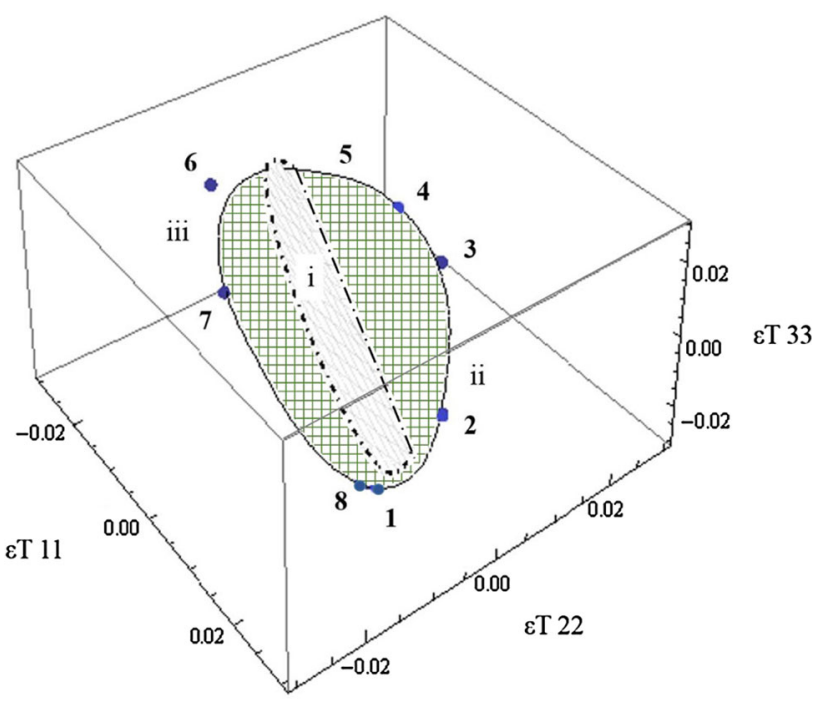

- Data i. Normal to anisotropic ( $\mathrm{S}$ as)

ii. Normal to isotropic ( $\mathrm{S}$ iso)

iii. Proposed evolution $(\mathrm{S} r)$

Fig. $73 \mathrm{D}$ map of the normal transformation strains for the rolled specimen at $\xi=70 \%$.

equation still lie on the locus that results from the isotropic prediction. Still, the configuration for $\dot{\varepsilon}_{r}^{t}$ falls back to $\dot{\varepsilon}_{i s o}^{t}$ if it is assumed that $a^{\varepsilon}=0$. Thus, it is shown that $\dot{\boldsymbol{\varepsilon}}_{r}^{t}$ is a general inclusive form. 
Table 3 Calibrated material parameters for the evolution equation (rolled specimen)

\begin{tabular}{lr}
\hline Identified parameter & \multicolumn{1}{l}{ Value } \\
\hline$a_{o}^{\varepsilon}$ & -0.0953 \\
$c^{\varepsilon}$ & 0.0814 \\
$F^{\varepsilon}$ & 0.0339 \\
$\omega_{o}^{\varepsilon}$ & -0.0373 \\
\hline
\end{tabular}

A notable conclusion drawn from the results of the micromechanics simulations is the strong effect of texture on the anisotropy of transformation strains. The point 7 in Fig. 6a corresponds to the response of the rolled specimen under uniaxial compression in the transverse direction of rolling (2-2 axis). Whereas an isotropic specimen would show a positive strain in the lateral direction (1-1 axis), this sample shows almost zero strain, and actually negative. The transformation strains corresponding to this point are as follows:

$-1.86 \%$ in the transverse direction;

$-0.05 \%$ in the rolling direction; and

$1.89 \%$ in the direction perpendicular to the rolling plane.

On the contrary, the transformation strain values for tension in the transverse direction, respective to the loading case 3 , are as follows:

$2.34 \%$ in the direction of $90^{\circ}$;

$-0.77 \%$ in the rolling direction; and

$-1.59 \%$ in the direction perpendicular to rolling.

These results are consistent with the observation that in copper-based alloys, martensitic transformation occurs with a small volume change. The obtained transformation strains are the result of the activated system of variants, governed by the conjunction of the loading path with the texture effect. A similar anisotropic behavior of transformation strains is apparent in Fig. $6 \mathrm{~b}$ as well. The point 2 corresponds to the response of the drawn specimen under equibiaxial tension. As opposed to the equal evolution of transformation strains for an isotropic material, in this case the material response favors the evolution of strains along the drawing direction. It appears that the effect of texture is much more prominent in the resulting transformation strains of the material than in yield stresses. The proposed evolution law seems to be able to approach the effect of processing on the end material behavior under proportional loading.

It is shown that the expression for $\dot{\boldsymbol{\varepsilon}}_{r}^{t}$ is more accurate to capturing the transformation strain in comparison to $\dot{\boldsymbol{\varepsilon}}_{i s \boldsymbol{t}}^{t}$. For the case of the rolled specimen, the values for the material parameters considered are shown in Table 3.

\section{Conclusion}

A new transformation criterion in terms of stresses and strains suitable for accurately describing the transformation of SMAs has been developed and implemented. The mathematical expressions governing the criterion in terms of stresses are studied with respect to convexity and capturing random anisotropy in SMAs' transformation. Furthermore, an accurate evolution rule to govern the evolution of transformation strain has been formulated. It is a non-associated evolution rule which captures incompressibility and still the anisotropy in strains. The equations of the criterion and the evolution rule have been calibrated for a copper-based textured SMA (Cu-Zn-Al), using the results from simulations of proportional uniaxial and biaxial plane stress loading states. These simulations were achieved by utilizing the numerical results of a self-consistent micromechanical model on three polycrystal configurations: isotropic, rolled, and drawn. Further results of the model have been used to assess and establish the accuracy of the proposed anisotropic criterion and the related non-associated evolution rule. A good agreement has been obtained by comparing the micromechanical simulations to results provided by the new formulated macroscopic model that can be easily implemented in FE codes. Accordingly, the effects of asymmetry and anisotropy of SMAs' behavior can be accounted for structural design of SMA actuators.

A key capability of the procedure used in this work to formulate and to calibrate the new transformation criterion and evolution equation is to establish a link between the processing conditions of a material and the final resulting macroscopic anisotropy of the overall behavior. Knowing the texture and the transformation parameters of an SMA polycrystal as a result of processing and passing through the micromechanical model, the macroscopic behavior is simulated and then the anisotropic effect is captured without the requirement of a large experimental database of multiaxial loadings.

The use of this new criterion, combined with a thermodynamical model, could extend the design capabilities of structures with highly textured SMAs. For such cases, the simulation of non-proportional loadings will be addressed in a future work.

Acknowledgments The authors would like to acknowledge Drs. Darren Hartl and Björn Kiefer for motivating parts of the discussion that led this work to its final form, as well as for their support and input. The support of the NSF International Institute of Multifunctional Materials for Energy Conversion (IIMEC), award \#0841082, is acknowledged. The gratitude of the authors is also extended to Dr. Jean-Sébastien Lecomte for plotting the pole figures. 
Fig. 8 Pole

figures corresponding to a isotropic specimen, $\mathbf{b}$ rolled specimen, and $\mathbf{c}$ drawn specimen

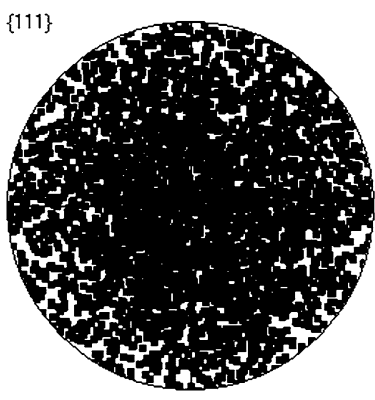

(a)

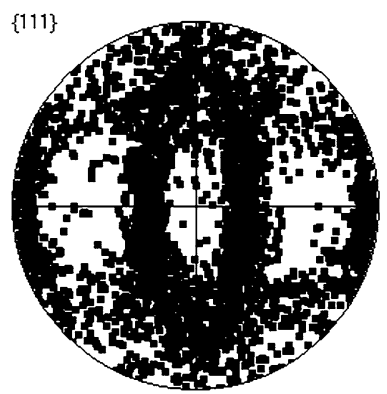

(b)

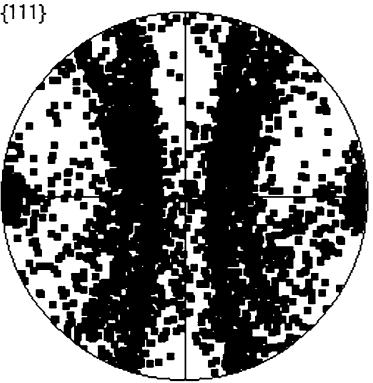

(c)

\section{Appendix 1: Transformation Rules of Stress Space}

The rotation matrices $\boldsymbol{R}_{z}, \boldsymbol{R}_{\boldsymbol{x}}, \boldsymbol{R}_{\boldsymbol{p}}$ appearing in (11) are found as such:

$$
\begin{aligned}
& \boldsymbol{R}_{z}=\left(\begin{array}{ccc}
\cos \theta_{z} & -\sin \theta_{z} & 0 \\
\sin \theta_{z} & \cos \theta_{z} & 0 \\
0 & 0 & 1
\end{array}\right), \\
& \boldsymbol{R}_{\boldsymbol{x}}=\left(\begin{array}{ccc}
1 & 0 & 0 \\
0 & \cos \theta_{x} & -\sin \theta_{x} \\
0 & \sin \theta_{x} & \cos \theta_{x}
\end{array}\right) \text { and } \\
& \boldsymbol{R}_{\boldsymbol{p}}=\left(\begin{array}{ccc}
\cos \theta_{p}+u_{1} & u_{1}-v_{1} & u_{1}+v_{1} \\
u_{1}+v_{1} & \cos \theta_{p}+u_{1} & u_{1}-v_{1} \\
u_{1}-v_{1} & u_{1}+v_{1} & \cos \theta_{p}+u_{1}
\end{array}\right), \\
& \text { where } u_{1}=\frac{1-\cos \theta_{p}}{3} \text { and } v_{1}=\frac{\sin \theta_{p}}{\sqrt{3}} .
\end{aligned}
$$

The tensor $\boldsymbol{R}_{\boldsymbol{\sigma}}$ is found finally as

$$
\boldsymbol{R}_{\sigma}=\left(\begin{array}{ccc}
\left(\begin{array}{ccc}
R_{11} & 0 & 0 \\
0 & R_{21} & 0 \\
0 & 0 & R_{31}
\end{array}\right) & \left(\begin{array}{ccc}
0 & l_{12} & 0 \\
0 & 0 & 0 \\
0 & 0 & 0
\end{array}\right) & \left(\begin{array}{ccc}
0 & 0 & l_{13} \\
0 & 0 & 0 \\
0 & 0 & 0
\end{array}\right) \\
\left(\begin{array}{ccc}
0 & 0 & 0 \\
l_{12} & 0 & 0 \\
0 & 0 & 0
\end{array}\right) & \left(\begin{array}{ccc}
R_{12} & 0 & 0 \\
0 & R_{22} & 0 \\
0 & 0 & R_{32}
\end{array}\right) & \left(\begin{array}{ccc}
0 & 0 & 0 \\
0 & 0 & l_{23} \\
0 & 0 & 0
\end{array}\right) \\
\left(\begin{array}{lll}
0 & 0 & 0 \\
0 & 0 & 0 \\
l_{13} & 0 & 0
\end{array}\right) & \left(\begin{array}{ccc}
0 & 0 & 0 \\
0 & 0 & 0 \\
0 & l_{23} & 0
\end{array}\right) & \left(\begin{array}{ccc}
R_{13} & 0 & 0 \\
0 & R_{23} & 0 \\
0 & 0 & R_{33}
\end{array}\right)
\end{array}\right),
$$

where $R_{i j}$ are found in (9).

\section{Appendix 2: Pole Figures for the Polycrystals}

See Fig. 8.

\section{References}

1. Bouvet C, Calloch S, Lexcellent C (2004) A phenomenological model for pseudoelasticity of shape memory alloys under multiaxial proportional and nonproportional loadings. Eur J Mech A Solids 23(1):37-61. doi:10.1016/j.euromechsol.2003.09.005

2. Zhu Y, Dui G (2010) A macro-constitutive model of polycrystalline NiTi SMAs including tensile-compressive asymmetry and torsion pseudoelastic behaviors. Int J Eng Sci 48(12):2099-2106. doi:10.1016/j.ijengsci.2010.04.002

3. Patoor E, El Amrani M, Eberhardt A, Berveiller M (1995) Determination of the origin for the dissymmetry observed between tensile and compression tests on shape memory alloys. J Phys IV C2:495-500

4. Šittner P, Novák V (2000) Anisotropy of martensitic transformations in modeling of shape memory alloy polycrystals. Int $\mathrm{J}$ Plast 16(10-11):1243-1268. doi:10.1016/S0749-6419(00)000097

5. Grolleau V, Louche H, Delobelle V, Penin A, Rio G, Liu Y, Favier D (2011) Assessment of tension-compression asymmetry of NiTi using circular bulge testing of thin plates. Scr Mater 65(4):347-350. doi:10.1016/j.scriptamat.2011.05.003

6. Sittner P, Heller L, Pilch J, Sedlak P, Frost M, Chemisky Y, Duval A, Piotrowski B, Ben Zineb T, Patoor E, Auricchio F, Morganti S, Reali A, Rio G, Favier D, Liu Y, Gibeau E, Lexcellent C, Boubakar L, Hartl D, Oehler S, Lagoudas D, Humbeeck JV (2009) Roundrobin SMA modeling. In: ESOMAT 2009-8th European symposium on martensitic transformations, EDP Sciences, Les Ulis, France, p 08001. doi:10.1051/esomat/ 200908001

7. Taillard K, Chirani SA, Calloch S, Lexcellent C (2008) Equivalent transformation strain and its relation with martensite volume fraction for isotropic and anisotropic shape memory alloys. Mech Mater 40(4-5):151-170. doi:10.1016/j.mechmat.2007.07.005

8. Taillard K (2006) Étude du comportement thermomécanique des alliages à mémoire de forme sous sollicitations multiaxiales complexes. Phd thesis, École Normale Supérieure de Cachan

9. Auricchio F, Taylor RL, Lubliner J (1997) Shape-memory alloys: macromodelling and numerical simulations of the superelastic behavior. Comput Methods Appl Mech Eng 146(3-4):281-312. doi:10.1016/S0045-7825(96)01232-7

10. Bodaghi M, Damanpack A, Aghdam M, Shakeri M (2014) A robust three-dimensional phenomenological model for 
polycrystalline smas: analytical closed-form solutions. Int J Eng Sci 82:1-21. doi:10.1016/j.ijsolstr.2014.01.0273

11. Boyd J, Lagoudas D (1996) A thermodynamical constitutive model for shape memory materials. Part I. The monolithic shape memory alloy. Int J Plast 12(6):805-842. doi:10.1016/S07496419(96)00030-7

12. Lagoudas D, Hartl D, Chemisky Y, Machado L, Popov P (2012) Constitutive model for the numerical analysis of phase transformation in polycrystalline shape memory alloys. Int $\mathrm{J}$ Plast 32-33:155-183. doi:10.1016/j.ijplas.2011.10.009

13. Patoor E, Lagoudas DC, Entchev PB, Brinson LC, Gao X (2006) Shape memory alloys, Part I: General properties and modeling of single crystals. Mech Mater 38(5--6):391-429. doi:10.1016/j. mechmat.2005.05.027

14. Chemisky Y, Duval A, Patoor E, Ben Zineb T (2011) Constitutive model for shape memory alloys including phase transformation, martensitic reorientation and twins accommodation. Mech Mater 43(7):361-376. doi:10.1016/j.mechmat.2011.04.003

15. Lexcellent C, Vivet A, Bouvet C, Calloch S, Blanc P (2002) IUTAM symposium on mechanics of martensitic phase transformation in solids. Springer

16. Peultier B, Ben Zineb T (2008) A simplified micromechanical constitutive law adapted to the design of shape memory applications by finite element methods. Mater Sci Eng A 481-482:384-388. doi:10.1016/j.msea.2007.05.117

17. Qidwai M, Lagoudas D (2000) On thermomechanics and transformation surfaces of polycrystalline NiTi shape memory alloy material. Int J Plast 16(10-11):1309-1343. doi:10.1016/S07496419(00)00012-7

18. Gibeau E, Laydi M, Lexcellent C (2010) Determination and transport of phase transformation yield surfaces for shape memory alloys. ZAMM - J Appl Math Mech/Zeitschrift für Angewandte Mathematik und Mechanik 90(7-8):595-604. doi:10. 1002/zamm.200900364

19. Hill R (1948) A theory of the yielding and plastic flow of anisotropic metals. Proc R Soc Lond 193:281-297

20. Tsai S, Wu E (1971) A general theory of strength for anisotropic materials. J Compos Mater 5:58-80

21. Patoor E, Eberhardt A, Berveiller M (1996) Micromechanical modelling of superelasticity in shape memory alloys. J Phys IV 6(C1):277-292

22. Boehlert C, Longanbach S, Bieler T (2008) Effect of thermomechanical processing on the creep behaviour of Udimet alloy 188. Philos Mag 88(5):641-664. doi:10.1080/14786430801944836

23. Somerday M, Comstock RJ, Wert JA (1997) A systematic analysis of transformation stress anisotropy in shape memory alloys. Philos Mag A 75(5):1193-1207. doi:10.1080/01418619708209851

24. Coleman B, Gurtin M (1967) Thermodynamics with internal variables. J Chem Phys 47(2):85-98

25. Lagoudas D (2008) Shape memory alloys-modeling and engineering applications. Springer, Berlin

26. Coleman B, Noll W (1963) The thermodynamics of elastic materials with heat conduction and viscosity. Arch Ration Mech Anal 13:167

27. Lubliner J (1972) On the thermodynamic foundations of nonlinear solid mechanics. Int J Non-linear Mech 7:237-254

28. Lemaitre J, Chaboche JL (2002) Mechanics of solid materials. Cambridge University Press. http://books.google.co.in/books/about/ Mechanics_of_Solid_Materials.html?id=YfHTNHfjqXoC\&pgis=1

29. Simo J, Hughes T (1998) Computational inelasticity. http://books. google.fr/books/about/Computational_Inelasticity.html?id=ftL2AJ L8OPYC\&pgis $=1$

30. Karafillis A, Boyce M (1993) A general anisotropic yield criterion using bounds and a transformation weighting tensor. J Mech Phys Solids 12:1859-1886
31. Boyd SP, Vandenberghe L (2004) Convex optimization. Cambridge University Press, Cambridge

32. Rockafellar RT (1970) Convex analysis. Princeton University Press, Princeton

33. Depriester D, Maynadier A, Lavernhe-Taillard K, Hubert O (2014) Thermomechanical modelling of a NiTi SMA sample submitted to displacement-controlled tensile test. Int J Solids Struct 51(10):1901-1922. doi:10.1016/j.ijsolstr.2014.01.0273

34. Meraghni F, Chemisky Y, Piotrowski B, Echchorfi R, Bourgeois N, Patoor E (2014) Parameter identification of a thermodynamic model for superelastic shape memory alloys using analytical calculation of the sensitivity matrix. Eur J Mech A Solids 45:226-237. doi:10.1016/j.euromechsol.2013.12.010

35. Merzouki T, Collard C, Bourgeois N, Ben Zineb T, Meraghni F (2010) Coupling between measured kinematic fields and multicrystal SMA finite element calculations. Mech Mater 42(1):72-95. doi:10.1016/j.mechmat.2009.09.003

36. Avril S, Bonnet M, Bretelle AS, Grédiac M, Hild F, Ienny P, Latourte F, Lemosse D, Pagano S, Pagnacco E, Pierron F (2008) Overview of identification methods of mechanical parameters based on full-field measurements. Exp Mech 48(4):381-402. doi:10.1007/s11340-008-9148-y

37. Sadjadpour A, Bhattacharya K (2007) A micromechanics inspired constitutive model for shape-memory alloys: the one-dimensional case. Smart Mater Struct 16(1):S51

38. Calloch S, Taillard K, Arbab Chirani S, Lexcellent C, Patoor E (2006) Relation between the martensite volume fraction and the equivalent transformation strain in shape memory alloys. Mater Sci Eng A 438-440:441-444. doi:10.1016/j.msea.2005.12.072

39. Mehrabi R, Andani MT, Elahinia M, Kadkhodaei M (2014) Anisotropic behavior of superelastic NiTi shape memory alloys; an experimental investigation and constitutive modeling. Mech of Mater 77:110-124. doi:10.1016/j.mechmat.2014.07.006

40. Hartl DJ, Mooney JT, Lagoudas DC, Calkins FT, Mabe JH (2010) Use of a $\mathrm{Ni}_{60} \mathrm{Ti}$ shape memory alloy for active jet engine chevron application: II. Experimentally validated numerical analysis. Smart Mater Struct 19(1):015021. doi:10.1088/09641726/19/1/015021

41. Entemeyer D (1996) Etude micromécanique du comportement thermomécanique des alliages à mémoire de forme. $\mathrm{PhD}$, Université de Metz

42. Cherkaoui M, Sun Q, Song G (2000) Micromechanics modeling of composite with ductile matrix and shape memory alloy reinforcement. Int J Solids Struct 37(11):1577-1594. doi:10.1016/ S0020-7683(98)00332-1

43. Siredey N, Patoor E, Berveiller M, Eberhardt A (1999) Constitutive equations for polycrystalline thermoelastic shape memory alloys. Int J Solids Struct 36(28):4289-4315. doi:10.1016/S00207683(98)00196-6

44. Wechsler M, Lieberman D, Read T (1953) On the theory of the formation of martensite. Trans AIME J Metals 197:1503-1515

45. Bowles JS, Wayman CM (1972) The bain strain, lattice correspondences, and deformations related to martensitic transformations. Metall Trans 3(5):1113-1121. doi:10.1007/BF02642442

46. Kaufman L, Cohen M (1958) Thermodynamics and kinetics of martensitic transformations. Prog Metal Phys 7:165-246. doi:10. 1016/0502-8205(58)90005-4

47. Collard C, Ben Zineb T (2012) Simulation of the effect of elastic precipitates in SMA materials based on a micromechanical model. Compos Part B: Eng 43(6):2560-2576. doi:10.1016/j. compositesb.2012.03.015

48. Piotrowski B, Ben Zineb T, Patoor E, Eberhardt A (2012) Modeling of niobium precipitates effect on the $\mathrm{Ni}_{47} \mathrm{Ti}_{44} \mathrm{Nb}_{9}$ shape memory alloy behavior. Int J Plast 36:130-147. doi:10.1016/j. ijplas.2012.03.004 
49. Chemisky Y, Duval A, Piotrowski B, Ben Zineb T, Tahiri V, Patoor E (2009) Numerical tool for SMA material simulation: application to composite structure design. Smart Mater Struct 18(10):104012. doi:10.1088/0964-1726/18/10/104012

50. Arbab Chirani S, Aleong D, Dumont C, McDowell D, Patoor E (2003) Superelastic behavior modeling in shape memory alloys. J Phys IV 112:205-208

51. Bhattacharya K, Khon R (1996) Symmetry, texture and the recoverable strain of shape memory polycrystal. Acta Mater 44(2):529-542
52. Gall K, Sehitoglou H (1999) The role of texture in tensioncompression asymmetry in polycrystalline NiTi. Int J Plast 15:69-92

53. Qu J, Cherkaoui M (2006) Fundamentals of micromechanics of solids. Wiley, Hoboken. doi:10.1002/9780470117835

54. Lagoudas DC, Gavazzi AC, Nigam H (1991) Elastoplastic behavior of metal matrix composites based on incremental plasticity and the mori-tanaka averaging scheme. Comput Mech 8(3):193-203. doi:10.1007/BF00372689 